\title{
Neutralism and selectionism: the molecular clock
}

\author{
Francisco J. Ayala* \\ Department of Ecology and Evolutionary Biology, University of California, Irvine, CA 92697-2525, USA
}

Received 22 June 2000; accepted 25 September 2000

Received by G. Bernardi

\begin{abstract}
The neutrality theory predicts that the rate of molecular evolution will be constant over time, and thus that there is a molecular clock for timing evolutionary events. It has been observed that the variance of the rate of evolution is generally larger than expected according to the neutrality theory. Several modifications of the theory have been proposed to account for the 'overdispersion' of the molecular clock, by postulating effects attributed to generation-time, population size, slightly deleterious mutations, repair mechanisms, and the like. An extensive investigation of two proteins, glycerol-3-phosphate dehydrogenase (GPDH) and superoxide dismutase (SOD), manifests that none of these modifications can simultaneously account for the disparate patterns observed in both proteins. GPDH evolves very slowly in Drosophila species, but several times faster in mammals, other animals, plants, and fungi. SOD evolves very fast in Drosophila species and also in mammals, but much more slowly in other animals and still slower when plants and fungi are compared to one another, or to animals. (C) 2000 Elsevier Science B.V. All rights reserved.
\end{abstract}

Keywords: Molecular evolution; Rates of evolution; Glycerol-3-phosphate dehydrogenase; Superoxide dismutase; Neutrality theory of evolution; Generationtime hypothesis

\section{Introduction}

The hypothesis of the molecular clock of evolution emerged from early observations that the number of amino acid differences in a given protein appeared to be proportional to the time elapsed since the divergence of the organisms compared (Zuckerkandl and Pauling, 1962; 1965; Margoliash, 1963). This observation was later stated by Kimura (1983, p. 98) as an 'empirical principle': 'For each protein, the rate of evolution in terms of amino acid substitutions is approximately constant per year per site for various lines, as long as the function and tertiary structure of the molecule remain essentially unaltered'. The time constancy was accounted for with the hypothesis that many amino acid (and nucleotide) substitutions may be of little or no functional consequence and that, therefore, most substitutions that occur in evolution will be of this kind rather than involve amino acid replacements strongly

\footnotetext{
Abbreviations: CR, Central repeat region; CSP, Circumsporozoite protein; MSP-1, Merozoite surface antigen protein-1; MSP-2, Merozoite surface antigen protein-2; R1a, R1b, R2a; Nucleotide repeat 1a, 1b, 2a in Msp-1; RAT, Repeat allotype; RHR1, RHR2, RHR3, Repeat homology region $1,2,3$

* Tel.: +1-949-824-8293; fax: 1-949-824-2474.

E-mail address: fjayala@uci.edu (F.J. Ayala).
}

constrained by natural selection (Zuckerkandl and Pauling, 1965).

Kimura's neutrality theory of molecular evolution provided a mathematical formulation for the hypothesis of the molecular clock (Kimura, 1968; see also Kimura, 1969, 1983). New alleles arise in a species by mutation. If alternative alleles are neutral with respect to natural selection (i.e. do not modify the Darwinian fitness of their carriers), their frequencies will only change by accidental sampling errors from generation to generation, that is, by genetic drift. Rates of allelic substitution will be stochastically constant they will occur with a constant probability for a given protein. Kimura showed that the rate of substitution of adaptively equivalent ('neutral') alleles, $k$, is precisely the rate of mutation; $u$, of neutral alleles, $k=u$.

According to the neutrality theory, evolution at the molecular level consists for the most part of the gradual, random replacement of one allele by another that is functionally equivalent to the first. The theory assumes that favorable mutations occur, but are sufficiently rare that they have little effect on the overall evolutionary rate of nucleotide and amino acid substitutions. A large proportion of all possible mutants are deleterious, but these are eliminated or kept at very low frequencies by natural selection, with little or no consequence in the rates of molecular evolution. 
In order to account for the apparent observation that the rate of amino acid substitution was constant over time, the neutrality theory postulated that the neutral mutation rate of amino acids was constant over time (Kimura, 1968, 1969, 1983). Ohta and Kimura (1971) statistically tested the neutrality hypothesis under the assumption that the number of substitutions has a Poisson distribution, with mean $k t$ (where $t$ is the age of the lineage in years). The Poisson process predicts that the variance, $\mathrm{V}$, will be equal to the mean, $\mathrm{M}$, so that their ratio, $R=\mathrm{V} / \mathrm{M}=1$. Ohta and Kimura's (1971; see Kimura, 1983; Gillespie, 1991) investigation of three proteins ( $\alpha$ globin, $\beta$ globin and cytochrome $c$ ) concluded that $R \sim 2$ and suggested reasons why the clock might be somewhat 'overdisperssed,' that is, $R>1$. Langley and Fitch (1974; Fitch, 1976) conducted an investigation of the same three proteins plus fibrinopeptide $\mathrm{A}$ in 18 vertebrate species and statistically rejected the neutrality hypothesis for overall rates (comparisons among branches), for relative rates (comparisons among proteins within branches), and for the total process. The matter was considered settled by many after an extensive study by Gillespie (1989; see 1991). The value of $R$ for 20 proteins spread from 0.32 to $43.82 ; 12$ values were significantly greater than 1 , as was the average, $R=6.95$ (corrected value by using weights from replacement substitutions; uncorrected $R=8.26$ ).

A number of hypotheses have been proposed to account for the overdispersion of $R$ and these will be briefly examined below. I want first to summarize our observations concerning the evolution of two proteins, part of an extensive investigation precisely designed as a test of the molecular clock hypothesis.

\section{Evolution of glycerol-3-phosphate dehydrogenase}

The nicotinamide adenine dinucleotide (NAD)-dependent cytoplasmic GPDH (glycerol-3-phosphate dehydrogenase) plays a crucial role in metabolism because of its keystone position in the glycerophosphate cycle, which in Drosophila and other dipterans provides energy for flight in the thoracic muscles (O'Brien and MacIntyre, 1978; Bewley, 1983). In Drosophila melanogaster, the Gpdh gene consists of eight exons and produces three isozymes by differential splicing of the last three exons (Cook et al., 1988; Bewley et al., 1989; von Kalm et al., 1989). We have analyzed in my laboratory 768 nucleotides from exons 3-6, coding for 256 amino acids, in 27 dipteran species (Kwiatowski et al., 1997; Barrio and Ayala, 1997). The species studied are from two families: Tephritidae, represented by the medfly Ceratitis capitata; and Drosophilidae represented by three genera: Scaptodrosophila (one species), Chymomyza (two species), and Drosophila (23 species, classified within five subgenera: Dorsilopha, Drosophila, HirtoDrosophila, Sophophora, and Zaprionus). We have investigated the amino acid and nucleotide composition of the homologous GPDH region in nine other species, encompassing three mammalian orders (human, mouse, and rabbit), three metazoan phyla (the nematode Coenorhabditis elegans, plus arthropods and chordates, represented by the mammal and fly species already listed), and three multicellular kingdoms (the angiosperm Cuphea lanceolata, the yeasts Schizosaccharomyces pombe and Saccharomyces cerevisiae, and the animals already listed).

The rate of amino acid replacements is not constant, but rather seems to increase with the time elapsed. The average number of amino acid replacements per 100 residues (Table 1) is $0.8-1.2$ between species from different Drosophila groups or subgenera, which diverged 45-55 My ago, but 3.0 between species from different genera, which diverged only somewhat earlier ( $60 \mathrm{My}$ ago). When comparisons are made between the medfly Ceratitis capitata and the Drosophilids, the number of amino acid replacements becomes $9.4 \pm 0.1$, for a time elapsed only twice as long as between the Drosophila genera or subgenera. The corresponding rates of amino acid replacement are shown in Fig. 1 (left). The rate of amino acid replacements observed in Drosophila lineages is $0.9-1.1 \times 10^{-10} /$ site/year, a very slow rate (comparable to the rates of $0.25-1.7$ observed for histone proteins; Wilson et al., 1977). But when Ceratitis and Drosophila species are compared, the lineage rate

Table 1

Evolution of GPDH: amino acid replacements between increasingly divergent species ${ }^{\mathrm{a}}$

\begin{tabular}{lccc}
\hline Comparison & My & Mean & Rate \\
\hline Within Drosophila groups & $5-25$ & $0.0-0.8$ & $0-2.1$ \\
Between Drosophila groups & $45 \pm 10$ & $0.8 \pm 0.0$ & $0.9 \pm 0.0$ \\
Between Drosophila subgenera & $55 \pm 10$ & $1.2 \pm 0.0$ & $1.1 \pm 0.0$ \\
Between dipteran genera & $60 \pm 10$ & $3.0 \pm 0.1$ & $2.7 \pm 0.1$ \\
Between mammals & $70 \pm 15$ & $7.4 \pm 1.6$ & $5.3 \pm 1.1$ \\
Between dipteran families & $100 \pm 20$ & $9.4 \pm 0.1$ & $4.7 \pm 0.1$ \\
Between animal phyla & $650 \pm 100$ & $54.7 \pm 0.3$ & $4.2 \pm 0.0$ \\
Between kingdoms & $1100 \pm 200$ & $87.0 \pm 0.8$ & $4.0 \pm 0.1$ \\
\hline
\end{tabular}

\footnotetext{
a The species compared and taxonomic position are given in Ayala et al. (1996). The plus/minus values are crude estimates of error for My, but are standard deviations for mean and rate. Mean values are per 100 residues for differences between species; the rates are lineage values, in units of $10^{-10}$ amino acid replacements/site/year. Replacements are corrected according to Dayhoff (1978). The matrices of amino acid and nucleotide differences are given in Barrio and Ayala (1997); Kwiatowski et al. (1997).
} 

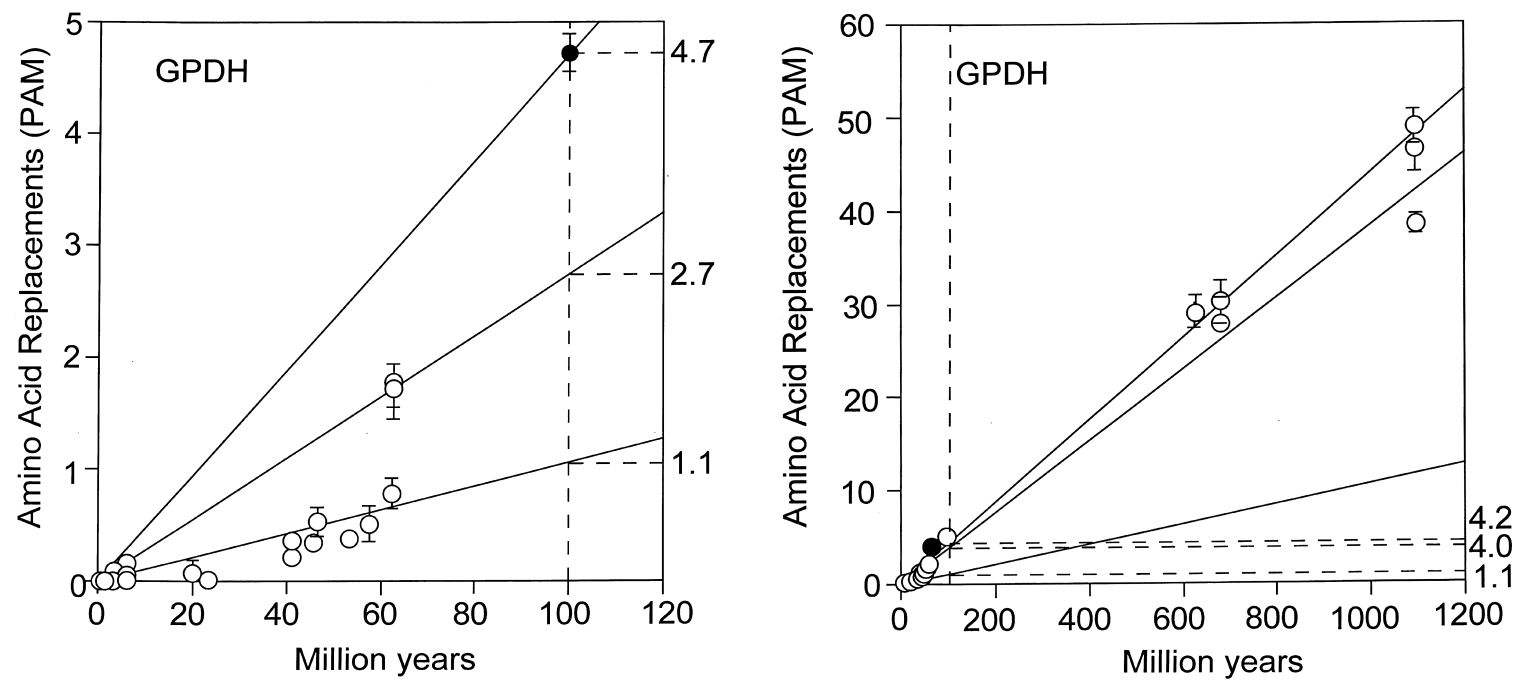

Fig. 1. Rate of molecular evolution in the protein GPDH. The rate numbers on the right refer to lineage changes in units of $10^{-10}$ amino acid replacements/site/ year. Left. The three lines correspond to the best-fitting rates for three sets of species considered: within the genus Drosophila (white circles, rate 1.1), between the genera Drosophila and Chymomyza (gray circles, rate 2.7), between the fruitfly families Drosophila and Ceratitis (black circle, rate 4.7). Right. The three lines correspond to the best-fitting rates for three sets of species: from different Drosophila subgenera (rate 1.1), animal phyla (flies vs. mammals vs. nematodes, rate 4.2), and kingdoms (animals vs. plants vs. fungi, rate 4.0). The black circle represents the rate of evolution for mammals (rate 5.3).

becomes five times greater, $4.7 \times 10^{-10} /$ site/year (slightly slower than the rates observed in some intracellular enzymes, such as $5.3 \times 10^{-10}$ for triosephosphate isomerase, or $6.7 \times 10^{-10}$ for cytochrome $c$ ).

The different rates of evolution displayed in Fig. 1 become even more disparate when we take into account that these rates apply to largely overlapping lineages. Consider, for example (Fig. 2), the evolution from node 3 to a Chymomyza species and a Drosophila species (say, $D$. melanogaster). The average rate of amino acid evolution is $2.7 \times 10^{-10}$ /site/year (Table 1 , line 4 ) over the 120 My of evolution separating these two species (60 My from node 3 to the Chymomyza species and $60 \mathrm{My}$ from node 3 to the
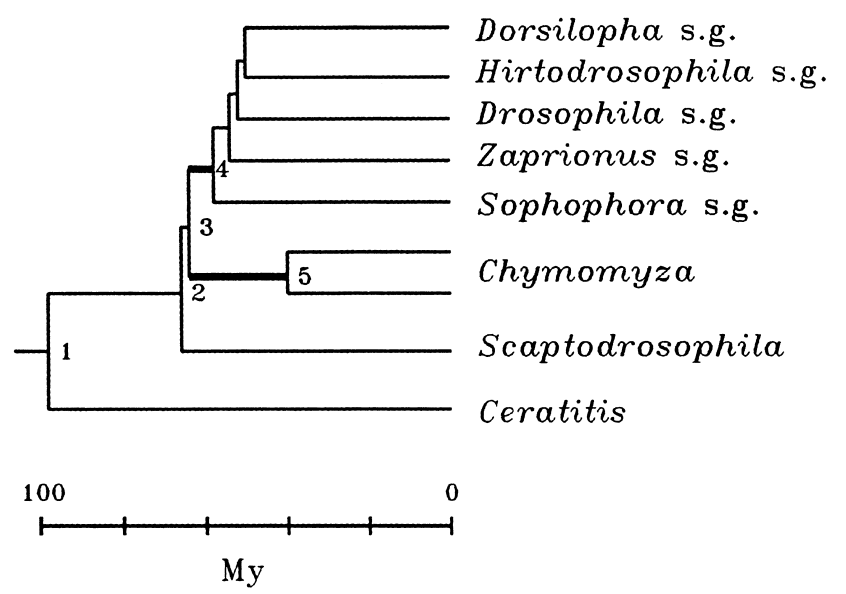

Fig. 2. Phylogeny of nine fruitfly species. The thicker branches between nodes 3 and 4 and between 3 and 5 indicate times with greatly accelerated evolution of GPDH. The time scale is based on data from Kwiatowski et al., (1994, 1997); Ayala et al., (1996).
Drosophila species). But during 100 of the $120 \mathrm{My}$ (55 My from node 4 to the Drosophila species and $45 \mathrm{My}$ from node 5 to the Chymomyza species), the rate of evolution is $0.9-1.1 \times 10^{-10}$ (Table 1, lines 2 and 3). Thus, the acceleration in rate of evolution could only have occurred over the $20 \mathrm{My}$ of evolution indicated by thick lines in Fig. 2 (between nodes 3 and 4 and between nodes 3 and 5). The rate of GPDH evolution during those $20 \mathrm{My}$ must have been $11 \times 10^{-10} /$ site/year, or more than 10 times faster than for the remainder $100 \mathrm{My}$, in order to give an average of $2.7 \times 10^{-10}$ over the $120 \mathrm{My}$. (The number of amino acid replacements between the Drosophila subgenera, or between the two Chymomyza species, is mostly 2, whereas between Chymomyza and Drosophila is 8 or 9.)

Similarly, the rate of GPDH divergence between the medfly Ceratitis and Drosophila is 4.7 times greater (Table 1, line 6, and Fig. 1, left) than between Drosophila species. But the accelerated rate of evolution could only have occurred for a fraction of the 200 My elapsed (between nodes 1 and 2 and between node 1 and Ceratitis), during which the rate of GPDH evolution must have been much more than five times faster than between Drosophila species.

Fig. 1 (right) displays the number of amino acid replacements against time for the dipteran lineages (highly compressed), as well as for three mammals (filled circle), three animal phyla, and three kingdoms. If we exclude the Drosophila lineages, the relationship is fairly linear, corresponding to a rate of amino acid replacement evolution of $4.0-5.3 \times 10^{-10} /$ site/year (Table 1 , lines $5,7,8$ ), fairly similar to the rate observed between Ceratitis and the Drosophilids $\left(4.7 \times 10^{-10} /\right.$ site/year, line 6$)$. At this scale, the rate of evolution of GPDH appears fairly clock-like. Nevertheless, 
this 'global' rate would yield very erroneous conclusions if it were used for timing events or for deciding branching topologies in the evolution of dipterans. It can be seen in Table 1 that the apparent rate of amino acid replacements is fairly constant for comparisons between species diverged $>70 \mathrm{My}$ ago (i.e. $4.0-5.3 \times 10^{-10} / \mathrm{site} / \mathrm{year}$ ), but this is $2-$ 5 times as large as between species diverged $<60$ My ago.

\section{Evolution of superoxide dismutases}

The superoxide dismutases (SOD) are abundant enzymes in aerobic organisms, protecting the cell against the harmfulness of free oxygen radicals (Fridovich, 1986). These enzymes have active centers that contain either iron or manganese, or both copper and zinc (Fridovich, 1986). The $\mathrm{Cu}, \mathrm{Zn}$ SOD is a well-studied protein, found in eukaryotes but also in some bacteria. The amino acid sequence is known in many organisms, plant, animals, fungi, and bacteria (Kwiatowski et al., 1991; Smith and Doolittle, 1992; Fitch and Ayala, 1994; Ayala et al., 1996).

The $\mathrm{Cu}-\mathrm{Zn} \mathrm{SOD}$ of $D$. melanogaster is a dimer molecule consisting of two identical polypeptide subunits associated with two $\mathrm{Cu}^{2+}$ and two $\mathrm{Zn}^{2+}$ per molecule. Each subunit has a molecular weight of 15,750 daltons and consists of 151 amino acids, the same as in other fruitfly species. The Drosophila Sod gene consists of two exons, separated by an intron 300-700 bp in length, located between codons 22 and 23. In Chymomyza, ScaptoDrosophila, and Ceratitis, there is an additional short intron $(<100 \mathrm{bp})$ between codons 95 and 96 (Kwiatowski et al., 1992a,b, 1994). We have sequenced the Sodgene in 27 fruitfly species, the same set (with two inconsequential differences) sequenced for
Table 2

Evolution of SOD: amino acid replacements between increasingly divergent species ${ }^{\mathrm{a}}$

\begin{tabular}{lccr}
\hline Comparison & \multicolumn{1}{l}{ My } & \multicolumn{1}{l}{ Mean } & \multicolumn{1}{l}{ Rate } \\
\hline Within Drosophila groups & $5-25$ & $0.0-11.4$ & $0-22.7$ \\
Between Drosophila groups & $45 \pm 10$ & $15.0 \pm 0.2$ & $16.6 \pm 0.2$ \\
Between Drosophila subgenera & $55 \pm 10$ & $17.8 \pm 0.3$ & $16.2 \pm 0.3$ \\
Between dipteran genera & $60 \pm 10$ & $21.4 \pm 0.2$ & $17.8 \pm 0.2$ \\
Between mammals & $70 \pm 15$ & $24.0 \pm 0.4$ & $17.2 \pm 0.3$ \\
Between dipteran families & $100 \pm 20$ & $31.8 \pm 0.6$ & $15.9 \pm 0.3$ \\
Between animal phyla & $650 \pm 100$ & $68.3 \pm 0.9$ & $5.3 \pm 0.1$ \\
Between kingdoms & $1100 \pm 200$ & $72.5 \pm 1.2$ & $3.3 \pm 0.1$ \\
\hline
\end{tabular}

${ }^{\text {a }}$ Species compared and other conventions are the same as in Table 1.

Gpdh (Kwiatowski et al., 1992a,b, 1994; Barrio and Ayala, 1997).

The data are summarized in Table 2. Fig. 3 (left) gives the Sod rate of amino acid evolution for the same set of dipteran species compared in Fig. 1. The rate of amino replacement is fairly linear over time, about $16-18 \times 10^{-10}$ amino acid replacements/site/year, although there are some conspicuously divergent points (see Fig. 3, left). The linearity of SOD evolution in these dipteran lineages contrasts with the sharp increase observed earlier in GPDH evolution when Chymomyza or Ceratitis are compared with the Drosophila,rather than Drosophila species with each other.

Fig. 3 (right) displays the SOD amino acid replacements for the same set of species investigated for GPDH in Fig. 1 (right) (except that the plant is now Ipomoea batatas rather than Cuphea lanceolata). As noted earlier (Ayala, 1986; but see also Lee et al., 1985), the rate of evolution of SOD seems to slow down as the lineages compared become
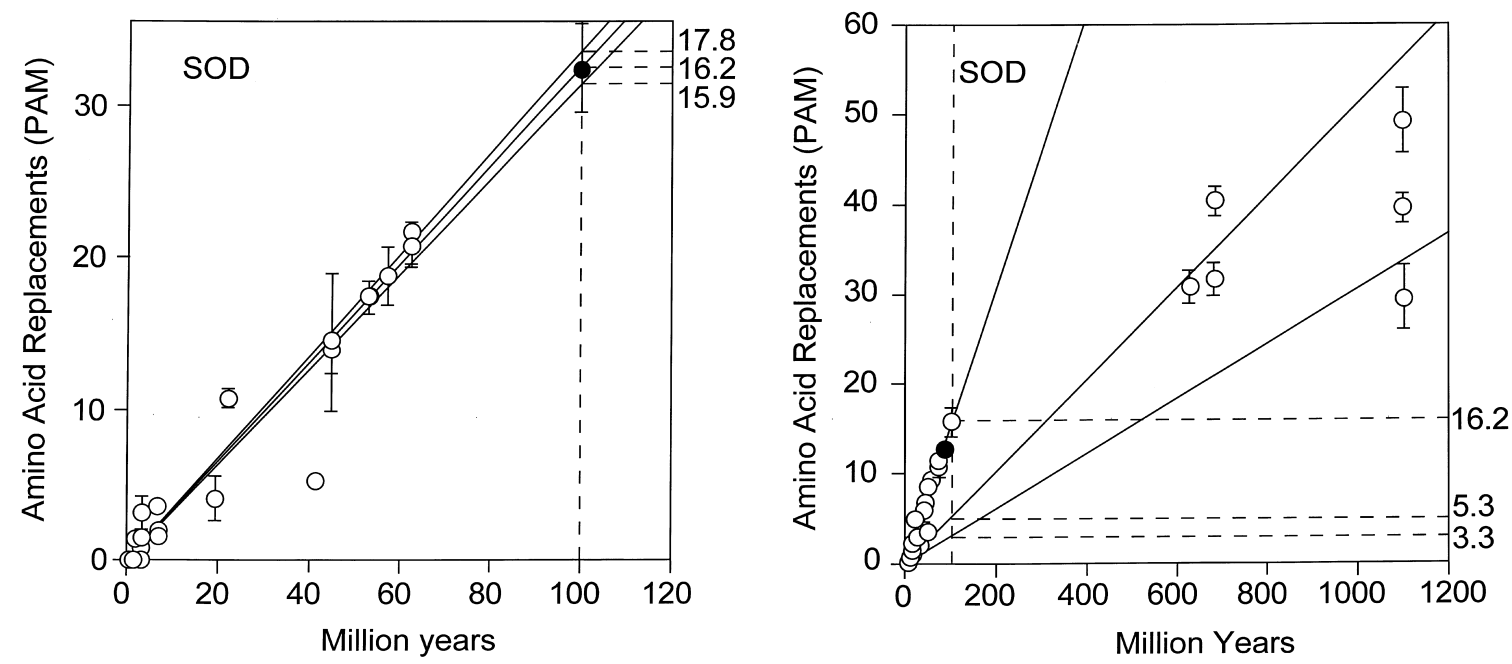

Fig. 3. Rate of molecular evolution of the protein $\mathrm{Cu}, \mathrm{Zn} \mathrm{SOD}$. The rate numbers on the right refer to lineage changes in units of $10^{-10}$ amino acid replacements/ site/year. Left. The three lines correspond to the best-fitting rates for the same three sets of species as in Fig. 1. The three rates (15.9, 16.2, and 17.8) are very similar to one another, in contradistinction to the disparate rates of GPDH for the same sets of species. Right. The three lines correspond to the best-fitting rates for three sets of species: from different Drosophila subgenera (rate 16.2), animal phyla (rate 5.3), and kingdoms (rate 3.3). These three rates are disparate and are much higher for species recently diverged (16.2, Drosophila) than for those that diverged long ago (3.3, for kingdoms), just the opposite to the shift occurring in GPDH. 
increasingly remote. This state of affairs is precisely the reverse of the pattern observed in GPDH, where the rate of amino acid replacement is slower when the species compared are closely related.

\section{Discussion}

The contrasting patterns of SOD and GPDH evolution cannot be attributed to distinctive characteristics of particular taxa, since the same set of species are compared for each enzyme (with the inconsequential exception that the plant species is Cuphea lanceolata for GPDH, but a different angiosperm, Ipomoea batatas for SOD). For the same reason, the rate fluctuations cannot be attributed to telescoping consequences of the different time intervals. The SOD rate is much faster for recently diverged taxa than for those that diverged long ago $\left(16.2 \times 10^{-10}\right.$ for Drosophila versus $3.3 \times 10^{-10}$ between kingdoms), but for GPDH it is exactly the other way around $\left(1.1 \times 10^{-10}\right.$ for Drosophila versus $4.0 \times 10^{-10}$ between kingdoms). Moreover, the mammal rate is similar to the Drosophila rate in SOD, but much faster in GPDH. The generation-time hypothesis (Wu and Li, 1985; Gaut et al., 1992; Li et al., 1996) cannot account for the erratic patterns of GPDH and SOD. This hypothesis assumes that rates of evolution will be increased in organisms with shorter generation times. But the set of species analyzed is, as noted, the same for GPDH and for SOD. It should also be noted that we have used a variety of algorithms for correcting for overlapping and back substitutions. These various algorithms all yield the same results, except for trivial numerical differences (Kwiatowski et al., 1992a, b, 1994, 1997; Barrio and Ayala, 1997).

Several modifications of the neutral theory have been proposed seeking to account for the excess variance of the molecular clock. Five biological variables have been invoked:

1. Generation time. Shorter generation time will accelerate the clock because it shortens the time to fix new mutations. It has been noted, for example, that the rate of molecular evolution is faster in rodents than in cows or primates (Gu and Li, 1992; Ohta, 1993).

2. Population size. A larger population size will slow the clock, because it increases the time required to fix new mutations. It has been suggested that population size and generation time are often inversely related (there are more mice than elephants), which thus might cancel out, and yield approximate constant rates over time (Ohta, 1972; Kimura, 1983). But this inverse relationship is far from general. Several species of Proechimysspiny rats in Venezuela and of Spalax mole rats in Israel have narrow ecological niches and small population sizes, while common rats and mice are cosmopolitan and several orders of magnitude more numerous. Drosophila willistoni is exceedingly numerous throughout the Amer- ican topics, while its sibling species, D. insularis, is restricted to two small islands in the Caribbean, where it is much rarer than $D$. willistoni; surely the population size of the two species differs by many orders of magnitude (and there is no reason to think that matters were different throughout the history of these two species). Yet, rates of molecular evolution among rodent species or among Drosophila species do not differ in patterns consistent with their differences in population size.

3. Slightly deleterious mutations. This hypothesis was proposed by Ohta $(1973,1974)$ and is called 'the model of effectively neutral mutations' by Kimura (1983, p. 240). Such mutations behave as neutral in small populations, but will be selected against in large populations. If selection coefficients against mutations follow an exponential distribution, the rate of substitution is inversely proportional to the effective population size of the species (Ohta, 1977).

4. Species-characteristic differences in polymerases or other biological properties that affect the fidelity of DNA replication, and hence the incidence of mutations, neutral or not (Britten, 1986). Without any specific knowledge about each particular species, this hypothesis simply says that molecular evolutionary rates will differ among species. But this hypothesis predicts that differences between species will be consistent across different genes, a prediction that does not hold for the combined cases of GPDH and SOD.

5. Changes in the function of a protein as evolutionary time proceeds. This might particularly be expected in the case of gene duplication, when one or both duplicates evolve new functions and hence an accelerated rate of evolution might be expected. Accelerated evolution following duplication has been observed, for example, in the globin genes (Goodman, 1976; Goodman et al., 1988). Except when postulated in association with gene duplication, this hypothesis is a special case of the general claim for a predominant role of natural selection in molecular evolution.

The covarion hypothesis (Fitch and Markowitz, 1970) becomes helpful to account for the reduction in rate of evolution that obtains in some proteins when the species compared become increasingly remote, as in the case of SOD (Fitch and Ayala, 1994). But the covarion model cannot be extended to GPDH, whose rate of evolution increases as the organisms compared become more remote (Ayala, 1997). Similarly, the combined consideration of GPDH and SOD evolution in the same set of species excludes any of the other proposed modifications of the neutrality theory listed above. The hypothesis of the generation-time effect cannot account for the divergent patterns of evolution of both GPDH and SOD, since the same set of species are compared in both cases, and thus identical generation times have been involved at all times in the evolution of these species. Similarly, the postulates of vari- 
Table 3

Rates of evolution of GPDH and SOD and estimates of divergence time derived from the Drosophila rate (Ayala, 1997) ${ }^{\mathrm{a}}$

\begin{tabular}{|c|c|c|c|c|c|c|}
\hline \multirow[t]{2}{*}{ Taxa compared } & \multicolumn{2}{|c|}{ Rate of evolution } & \multicolumn{2}{|c|}{ Normalized rate } & \multicolumn{2}{|c|}{ Clock estimates, My } \\
\hline & GPDH & SOD & GPDH & SOD & GPDH & SOD \\
\hline Drosophila subgenera & 1.1 & 16.2 & 1.0 & 1.0 & 55 & 55 \\
\hline Mammal orders & 5.3 & 17.2 & 4.8 & 1.1 & 340 & 74 \\
\hline Dipteran families & 4.7 & 15.9 & 4.3 & 1.0 & 470 & 98 \\
\hline Animal phyla & 4.2 & 5.3 & 3.8 & 0.33 & 2,500 & 211 \\
\hline Kingdoms & 4.0 & 3.3 & 3.6 & 0.20 & 3,990 & 224 \\
\hline
\end{tabular}

${ }^{a}$ The rate of evolution is in units of $10^{-10}$ amino acid replacements/site/year. The normalized rate is relative to the rate between the Drosophila subgenera. The clock estimates of time divergence use the average amino acid replacements of the particular organisms and assumes that the replacement rate is constant, so that the protein evolves as a molecular clock that ticks at the Drosophila rate.

able population size, slightly deleterious mutations or differences in the efficiency of repair mechanisms may be adjusted to account for the evolution of one or the other protein, GPDH and SOD, but not for both, without stretching ad hoc their elasticity to make the molecular clock hypothesis universally applicable to any possible empirical state of affairs and, therefore, without any predictive power and untestable (Popper, 1959; Ayala, 1994).

I have noted (Ayala, 1997) that constraints occur in the evolution of GPDH in Drosophila, which considerably restrict the number of sites that can accept amino acid replacements and the particular replacements that can occur at each site. It remains obscure why greater constraints would occur in Drosophila than in the Chymomyza or Ceratitis lineages (or, indeed, in other animals, plants, and fungi). But, in any case, the issue is not whether biologically ascertainable processes are at work, which of course they are, in GPDH, SOD, or any other enzymes. The issue rather is whether the processes are of such regularity that some sort of molecular clock may be assumed. The stark contrast between the pattern of evolution of GPDH and SOD may be an aberration rather than representative of prevailing modes of protein evolution, since protein evolution seems so often to behave in a clock-like manner. But the congruence between observations and the clock predictions are often obtained due to the fact that the data collected do not have sufficient resolution to manifest likely discrepancies.

The operational risks of assuming that protein clocks are fairly reliable are made evident in Table 3 . The rate of GPDH evolution is nearly four times faster between animals and plants than between Drosophila species, whereas the rate of SOD evolution is five times slower. If we were to use the observed rate of Drosophila evolution to estimate the time of divergence between plants and animals, GPDH would yield an estimate of 3,990 My, SOD an estimate of $224 \mathrm{My}$, both grossly erroneous. The practical conclusions to be drawn are that (i) protein clocks should be used cautiously and weighed against any other available evidence, rather than considered decisive; (ii) several protein clocks should be used whenever feasible, particu- larly when important evolutionary events need to be determined (Wray et al., 1996; but see Ayala et al., 1998).

\section{References}

Ayala, F.J., 1986. On the virtues and pitfalls of the molecular evolutionary clock. J. Heredity 77, 226-235.

Ayala, F.J., 1994. On the scientific method, its practice and pitfalls. Hist. Phil. Life Sci. 16, 205-240.

Ayala, F.J., 1997. Vagaries of the molecular clock. Proc. Natl. Acad. Sci. USA 94, 7776-7783.

Ayala, F.J., Barrio, E., Kwiatowski, J., 1996. Molecular clock or erratic evolution? A tale of two genes. Proc. Natl. Acad. Sci. USA 93, 11729 11734.

Ayala, F.J., Rzhetsky, A., Ayala, F.J., 1998. Origin of the metazoan phyla: Molecular clocks confirm paleontological estimates. Proc. Natl. Acad. Sci. USA 95, 606-611.

Barrio, E., Ayala, F.J., 1997. Evolution of the Drosophila obscura species group inferred from the Gpdh and Sod genes. Mol. Phylogen. Evol. 7, 79-93.

Bewley, G.C., 1983. The genetic and epigenetic control of SN-glycerol-3phosphate dehydrogenase isozyme expression during the development of Drosophila melanogaster. In: Rattazzi, M.C., Scandalios, J.G., Whitt, G.S. (Eds.). Isozymes: Current Topics in Biological and Medical Research. Alan R. Liss, New York, pp. 33-62.

Bewley, G.C., Cook, J.L., Kusakabe, S., Mukai, T., Rigby, D.L., Chambers, G.K., 1989. Sequence, structure and evolution of the gene coding for SN-glycerol-3-phosphate dehydrogenase in Drosophila melanogaster. Nucleic Acids Res. 17, 8553-8567.

Britten, R.J., 1986. Rates of DNA sequence evolution differ between taxonomic groups. Science 231, 1393-1398.

Cook, J.L., Bewley, G.C., Shaffer, J.B., 1988. Drosophila-SN-glycerol-3phosphate dehydrogenase isozymes are generated by alternate pathways of RNA processing resulting in different carboxyl-terminal amino-acid sequences. J. Biol. Chem. 263, 10858-10864.

Dayhoff, M.D., 1978. Atlas of Protein Sequences and Structure. Natl. Biomed. Res. Found., Washington, DC.

Fitch, W.M., 1976. Molecular evolutionary clocks. In: Ayala, F.J. (Ed.). Molecular Evolution. Sinauer, Sunderland, MA, pp. 160-178.

Fitch, W.M., Ayala, F.J., 1994. The superoxide dismutase molecular clock revisited. Proc. Natl. Acad. Sci. USA 91, 6802-6807.

Fitch, W.M., Markowitz, E., 1970. An improved method for determining codon variability in a gene and its application to the rate of fixations of mutations in evolution. Biochem. Gen. 4, 579-593.

Fridovich, I., 1986. Superoxide dismutases. Adv. Enzymol. 58, 61-97.

Gaut, B.S., Muse, S.V., Clark, W.D., Clegg, M.T., 1992. Relative rates of 
nucleotide substitution at the $r b c \mathrm{~L}$ locus of monocotyledonous plants. J. Mol. Evol. 35, 292-303.

Gillespie, J.H., 1989. Lineage effects and the index of dispersion of molecular evolution. Mol. Biol. Evol. 6, 636-647.

Gillespie, J.H., 1991. The Causes of Molecular Evolution. Oxford University Press, New York.

Goodman, M., 1976. Protein sequences in phylogeny. In: Ayala, F.J. (Ed.). Molecular Evolution. Sinauer, Sunderland, MA, pp. 141-159.

Goodman, M., Pedwaydon, J., Czelusniak, J., Suzuki, T., Gotoh, T., Moens, L., Shishikura, F., Walz, D., Vinogradov, S., 1988. An evolutionary tree for invertebrate globin sequences. J. Mol. Evol. 27, 236-249.

Gu, X., Li, W.-H., 1992. Higher rates of amino acid substitution in rodents than in humans. Mol. Phylogenet. Evol. 1, 211-214.

Kimura, M., 1968. Evolutionary rate at the molecular level. Nature (Lond.) 217, 624-626.

Kimura, M., 1969. The rate of molecular evolution considered from the standpoint of population genetics. Proc. Natl. Acad. Sci. USA 63, 11811188.

Kimura, M., 1983. The Neutral Theory of Molecular Evolution. Cambridge University Press, Cambridge.

Kwiatowski, J., Hudson, R.R., Ayala, F.J., 1991. The rate of Cu,Zn superoxide dismutase evolution. Free Radic. Res. Commun. 12-13, 363-370.

Kwiatowski, J., Skarecky, D., Burgos, M., Ayala, F.J., 1992a. Structure and sequence of the $\mathrm{Cu}, \mathrm{Zn}$ superoxide dismutase gene of Chymomyza amoena: phylogeny of the genus and codon-use evolution. Insect Mol. Biol. 1, 3-13.

Kwiatowski, J., Skarecky, D., Ayala, F.J., 1992b. Structure and sequence of the $\mathrm{Cu} / \mathrm{Zn}$ Sod gene in the Mediterranean fruit-fly Ceratitis Capitata: Intron insertion/deletion and evolution of the gene. Mol. Phyl. Evol. 1, $72-82$.

Kwiatowski, J., Skarecky, D., Bailey, K., Ayala, F.J., 1994. Phylogeny of Drosophila and related genera inferred from the nucleotide sequence of the Cu,Zn Sod gene. J. Mol. Evol. 38, 343-353.

Kwiatowski, J., Krawczyk, M., Jaworski, M., Skarecky, D., Ayala, F.J., 1997. Erratic evolution of glycerol-3-phosphate dehydrogenase in Drosophila Chymomyza, and Ceratitis. J. Mol. Evol. 44, 9-22.

Langley, C.H., Fitch, W.M., 1974. An examination of the constancy of the rate of molecular evolution. J. Mol. Evol. 3, 161-177.

Lee, Y.M., Friedman, D.J., Ayala, F.J., 1985. Superoxide dismutase: an evolutionary puzzle. Proc. Natl. Acad. Sci. USA 82, 824-828.

Li, W.-H., Ellsworth, D.L., Kruchkal, J.K., Chang, B.H.-J., HewettEmmett, D., 1996. Rates of nucleotide substitution in primates and rodents and the generation time effect hypothesis. Mol. Phylogenet. Evol. 5, 182-187.

Margoliash, E., 1963. Primary structure and evolution of cytochrome $c$. Proc. Natl. Acad. Sci. USA 50, 672-679.

O'Brien, S.J., MacIntyre, R.J., 1978. Genetics and biochemistry of enzymes and specific proteins of Drosophila. In: Ashburner, M., Wright, T.R.F. (Eds.). The Genetics and Biology of Drosophila, Vol. 2a. Academic Press, New York, pp. 396-552.

Ohta, T., 1972. Population size and rate of evolution. J. Mol. Evol. 1, 305314

Ohta, T., 1973. Slightly deleterious substitutions in evolution. Nature 246, 96-98.

Ohta, T., 1974. Mutational pressure as the main cause of molecular evolution and polymorphism. Nature 252, 351-354.

Ohta, T., 1977. Extension to the neutral mutation random drift hypothesis. In: Kimura, M. (Ed.). Molecular Evolution and Polymorphism. National Institute of Genetics, Mishima, pp. 148-167.

Ohta, T., 1993. An examination of the generation time effect on molecular evolution. Proc. Natl. Acad. Sci. USA 90, 10676-10680.

Ohta, T., Kimura, M., 1971. On the constancy of the evolutionary rate of cistron. J. Mol. Evol. 1, 18-25.

Popper, K.R., 1959. The Logic of Scientific Discovery, Hutchinson, London.

Smith, M.W., Doolittle, R.F., 1992. Anomolous phylogeny involving the enzyme glucose-6-phosphate isomerase. J. Mol. Evol. 34, 175-184.

Wilson, A.C., Carlson, S.S., White, T.J., 1977. Biochemical evolution. Ann. Rev. Biochem. 46, 573-639.

Wray, G.A., Levinton, J.S., Shapiro, L.H., 1996. Molecular evidence for deep precambrian divergences among metazoan phyla. Science 274 , 568-573.

Wu, C.-I., Li, W.-H., 1985. Evidence for higher rates of nucleotide substitution in rodents than in man. Proc. Natl. Acad. Sci. USA 82, 17411745.

Zuckerkandl, E., Pauling, L., 1962. Molecular disease, evolution and genic heterogeneity. In: Kash, M., Pullman, B. (Eds.). Horizons in Biochemistry. Academic Press, New York, pp. 97-166.

Zuckerkandl, E., Pauling, L., 1965. Evolutionary divergence and convergence in proteins. In: Bryson, V., Vogel, H.J. (Eds.). Evolving Genes and Proteins. Academic Press, New York, pp. 97-166.

von Kalm, L., Weaver, J., DeMarco, J., MacIntyre, R.J., Sullivan, D.T., 1989. Structural characterization of the alpha-glycerol-3-phosphate dehydrogenase-encoding gene of Drosophila-melanogaster. Proc. Natl. Acad. Sci. USA 86, 5020-5024. 